\title{
Type II Secretory Phospholipase A2 Measurement
}

National Cancer Institute

\section{Source}

National Cancer Institute. Type I/ Secretory Phospholipase A2 Measurement. NCI

Thesaurus. Code C120663.

The determination of the type II secretory phospholipase A2 present in a sample. 